\title{
Object Substitution Without Reentry?
}

\author{
Vincent Di Lollo, James T. Enns, and Ronald A. Rensink \\ University of British Columbia
}

\begin{abstract}
G. Francis and F. Hermens (2002) used computer simulations to claim that many current models of metacontrast masking can account for the findings of V. Di Lollo, J. T. Enns, and R. A. Rensink (2000). They also claimed that notions of reentrant processing are not necessary because all of V. Di Lollo et al. 's data can be explained by feed-forward models. The authors show that G. Francis and F. Hermens's claims are vitiated by inappropriate modeling of attention and by ignoring important aspects of V. Di Lollo et al. 's results.
\end{abstract}

We note with interest Francis and Hermens's (2002) article, which purports to show that the findings reported by Di Lollo, Enns, and Rensink (2000) can be explained by other models of metacontrast masking. To buttress their claim, Francis and Hermens reported computer simulations showing that some of our results can be modeled by the theories of Bridgeman (1978), Francis (2000) and Weisstein (1968). This claim has a good deal of surface appeal because it is parsimonious. It argues that our results can be explained without recourse to the new concept of object substitution. Parsimony, however, is achieved at the cost of inappropriate modeling of attention and modeling an incomplete portion of our masking data. Here, we reiterate our original claim that reentrant modeling is necessary for explaining our findings. We do so by showing that a plausible case for the sufficiency of feed-forward processes has not been made by Francis and Hermens.

\section{Modeling of Attention}

Modeling of the effects of attention in Di Lollo et al. 's (2000) study was based on the large literature on set-size effects in visual perception (Duncan \& Humphreys, 1989; Eriksen,1995; Sperling, 1960; Treisman \& Gelade, 1980). Accordingly, set size was modeled as though it influenced the time required for attention to be deployed to the location of the target. The larger the set size, the longer it takes to find the target.

Current models of masking do not encompass attentional effects. This is one reason why they cannot account for Di Lollo et al. 's (2000) data. Francis and Hermens (2002) acknowledged this but claimed that it is fairly trivial to extend those models to include attentional effects. They did so by

Vincent Di Lollo, James T. Enns, and Ronald A. Rensink, Department of Psychology, , Vancouver, BC, Canada.

This work was sponsored by Natural Sciences and Enginee-ring Research Council of Canada grants to Vincent Di Lollo, James T. Enns, and Ronald A. Rensink.

Correspondence may be addressed to: Vincent Di Lollo, Department of Psychology, University of British Columbia, 2136 West Mall, Vancouver, British Columbia V6T 1Z4 Canada. Email: vince.dilollo@ubc.ca. modeling attention as affecting the intensity of the mask. As set size is increased, the mask representation is assumed to become stronger whereas the target representation remains the same. We believe this modeling of attention to be inappropriate.

It has long been known that the strength of metacontrast masking is directly related to mask intensity (Alpern, 1953). It is, therefore, unsurprising that weakening the mask reduces the metacontrast effect in all extant models. There is no need to expand current models along these lines because they already include the effect of mask intensity. The question that Francis and Hermens (2002) did not address is why anyone should model attention in terms of mask intensity unless they were aware of Di Lollo et al. 's (2000) results and did not have any other convenient mechanism for reducing the magnitude of the metacontrast effect. In more general terms, there is a substantial difference between claiming that current models can account for some data and claiming that current models can account for those data with the addition of arbitrary and post hoc assumptions.

But problems remain even if one assumes that the intensity of the mask is indeed modulated by the distribution of attention. One problem pertains to the spatial resolution of attention. In the Di Lollo et al. (2000) experiment modeled by Francis and Hermens (2002), the mask consisted of four small dots at the corners of an imaginary square, and the target was a small ring drawn inside the imaginary square. The target-mask separation was only $10 \mathrm{~min}$ arc. If one postulates that attention can increase the intensity of the mask without producing a corresponding increment in the target, one must also assume that the spatial resolution of attention is at least as fine as the target-mask separation. This assumption is disconfirmed by evidence that the resolution of attention is measured not in minutes but in degrees of visual angle (He, Cavanagh, \& Intriligator, 1997; La Berge, 1990). It is, therefore, unrealistic to assume that in the Di Lollo et al. experiment attention could modulate the intensity of the mask without also modulating the intensity of the target in the same way. Needless to say, this further questions the validity of Francis and Hermens's simulations.

A third problem concerns the magnitude of the hypothesized attentional effects as compared with corresponding sensory effects. There is no question that the results modeled by Francis 
and Hermens (2002) can be obtained by varying the physical intensity of the mask over a wide range of values. What is questionable, however, is whether a corresponding range of perceived mask intensities can be obtained by varying focal attention alone, given a stimulus of fixed energy, as was done in our experiments. The plausibility of this proposal is of course easily testable, but Francis and Hermens failed to provide any evidence. The existing data of Spencer and Shuntich's (1970) study on the interaction of intensity and set size in masking by overlapping patterns suggest, if anything, that backward masking at stimulus onset asynchronies (SOAs) beyond about $100 \mathrm{~ms}$ is unaffected by mask intensity. Intensity seems to play its largest role in the narrow range of SOAs around the target display when masking by integration is at work.

For these and other related reasons, which include both a simulated mask many times more spatially extensive than our four-dot mask and evidence that perceived intensity is unaffected by focal attention (Eckstein, Shimozaki, \& Abbey, 2002), Francis and Hermens's (2002) modeling of the attentional effects in Di Lollo et al. 's (2000) study is unrealistic and wide of the mark.

\section{Modeling an Incomplete Set of Masking Data}

Two masking processes emerged from the Di Lollo et al. (2000) study: an early process affected by physical factors such as adapting luminance and a later process affected by attentional factors such as set size. The early process is in evidence only in light-adapted viewing. In contrast, the late process is also in evidence in dark-adapted viewing. This suggests that the early process may well be based on inhibitory contour interactions, which are known to occur in the light but are much reduced or absent in the dark (von Békésy, 1968). Our dark-adapted experiments were aimed at underscoring that distinction and at emphasizing that two sets of explanatory principles were required to provide a comprehensive account of all our findings. Francis and Hermens (2002) overlooked this distinction and maintained that all of our findings can be explained by a single set of principles.

Overlooking this critical distinction led Francis and Hermens (2002) to conclude that Weisstein's (1968) model, which is based on inhibitory contour interactions, could account for all our results. In fact, Weisstein's model can account for masking obtained under light-adapted but not under dark-adapted viewing conditions. The reasons are as follows. It is known that conventional metacontrast masking is much reduced or eliminated in dark-adapted viewing (Bischof \& Di Lollo, 1995). Inhibitory theories, such as Weisstein's, can explain such absence of masking on the grounds that whatever inhibitory processes, whether retinal or cortical, mediate metacontrast masking in light-adapted viewing are missing in dark-adapted viewing, hence masking cannot occur in the dark. However, the very principle (lack of inhibition in the dark) that permits inhibitory theories to explain why masking is absent in the dark prevents them from explaining Di Lollo et al. 's (2000) finding that under the same dark-adapted viewing condition, object-substitution masking is present in full force. Thus, Weisstein's model can certainly account for some of our results in light-adapted viewing. What it cannot do is account for our results in dark-adapted viewing because the type of inhibitory effects on which the model is predicated are known to be drastically reduced or absent in the dark. To be clear about this, the absence of masking in the dark (Bischof \& Di Lollo, 1995) and its presence (Di Lollo et al., 2000) cannot both be explained using the same set of principles. Yet, that is what Francis and Hermens attempted to do in their simulation of Weisstein's model.

\section{Concluding Comments}

This leads to our final point regarding Francis and Hermens's (2002) assertion that notions of reentrant processing are not necessary: Feed-forward principles can account for all our findings. This is not correct. Of the three theories modeled by Francis and Hermens, only Weisstein's (1968) is strictly feedforward, and we have pointed out ways in which it cannot account for all the data. But we disagree with Francis and Hermens's conclusion at a more fundamental level. It is known that neural pathways between brain regions are seldom, if ever, one way. If Area A sends signals to Area B, then Area B sends signals back to Area $A$. The reentrant pathways are massive and often contain more fibers than the corresponding ascending pathways. Given this brain architecture, reentrant processing should be regarded as the default principle on which to base our theories. To insist that, at least in the case of our findings, the default option should be feed forward is unrealistic. This is especially so because there are as yet no feed-forward models of which we are aware that can explain the conjoint findings of masking by common onset and the role of attention in masking by object substitution.

We appreciate Francis and Hermens's (2002) interest and value their comments. However, their suggestions as to how other models can account for our data must await a more valid and realistic approach.

\section{References}

Alpern, M. (1953). Metacontrast. Journal of the Optical Society of America, 43, 648-657.

Bischof, W. F. \& Di Lollo, V. (1995). Motion and metacontrast with simultaneous onset of stimuli. Journal of the Optical Society of America A, 12, 1623-1636.

Bridgeman, B. (1978). Distributed sensory coding applied to simulation of iconic storage and metacontrast. Bulletin of Mathematical Biology, 40, 605-623.

Di Lollo, V., Enns, J. T. \& Rensink, R. A. (2000). Competition for consciousness among visual events: The psychophysics of reentrant visual processes. Journal of Experimental Psychology: General, 129, 481-507.

Duncan, J. \& Humphreys, G. (1989). Visual search and stimulus similarity. Psychological Review, 96, 433-458. 
Eckstein, M. P., Shimozaki, S. S. \& Abbey, C. K. (2002). The footprints of visual attention in the Posner cueing paradigm revealed by classification images. Journal of Vision, 2, 25-45.

Eriksen, C. W. (1995). The flankers task and response competition: A useful tool for investigating a variety of cognitive problems. Visual Cognition, 2, 101-118.

Francis, G. (2000). Quantitative theories of metacontrast masking. Psychological Review, 107, 768-785.

Francis, G. \& Hermens, F. (2002). Comment on "Competition for Consciousness Among Visual Events: The Psychophysics of Reentrant Visual Processes" ( Journal of Experimental Psychology: General, 131, xxx-xxx.

He, S., Cavanagh, P. \& Intriligator, J. (1997). Attentional resolution. Trends in Cognitive Sciences, 1, 115-121.

La Berge, D. L. (1990). Attention. Psychological Science, 1, 156162 .

Spencer, T. J. \& Shuntich, R. (1970). Evidence for an interruption theory of backward masking. Journal of Experimental Psychology, $85,198-203$
Sperling, G. (1960). The information available in brief visual presentations. Psychological Monographs, 74(11, Whole No. 498).

Treisman, A. \& Gelade, G. (1980). A feature integration theory of attention. Cognitive Psychology, 12, 97-136.

von Békésy, G. (1968). Mach- and Hering-type lateral inhibition in vision. Vision Research, 8, 1483-1499.

Weisstein, N. (1968). A Rashevsky-Landahl neural net: Stimu-lation of metacontrast. Psychological Review, 75, 494-521.

Received: April 8, 2002

Revised: July 2, 2002

Accepted: July 2, 2002 